\title{
Altura de extracción de la muestra para evaluación de densidad básica y blancura en madera de Salix $s p$.
}

\author{
Sampling height for basic density and brightness evaluation in wood of Salix $s p$. \\ MARIA SILVINA VILLEGAS ${ }^{1}$, RAUL MARLATS ${ }^{2}$ \\ ${ }^{1}$ Cátedra de Dendrología, FCAF, UNLP, cc 31, 60 y 119, (CP 1900) La Plata, Bs. As., Argentina. \\ E-mail: silvillegas@ceres.agro.unlp.edu.ar \\ ${ }^{2}$ Cátedra de Silvicultura, FCAF, UNLP, cc 31, 60 y 119, (CP 1900) La Plata, Bs. As., Argentina. \\ E-mail: rmarlats@ceres.agro.unlp.edu.ar
}

\begin{abstract}
SUMMARY
The aim of the present work was to find the optimum sampling point representative for the whole tree, for basic density and brightness evaluation, in two Salix clones growing in two different sites of Delta of Paraná, Argentina. Thirty-two trees were sampled, aged 8 to 13 years old. Disks were taken at three heights, called "predictive sections" ( $1.30 \mathrm{~m}, 25 \%$ and $65 \%$ of commercial height). Radial variation was studied by sampling each wood disk in two opposite cardinal directions at three points of the radius $(33 \%, 66 \%$ and $100 \%$ of its length), amounting to 18 sampling positions in each tree. The parameter estimation of the whole tree and predictive sections was calculated as weighted mean values. The relationship between whole tree and predictive sections values was quantified using linear regression analysis. The breast height $(1.30 \mathrm{~m})$ was the best level for predicting American willow mean basic density and hybrid willow mean brightness of the whole tree, when the fittings obtained were considered. The $25 \%$ and $65 \%$ of commercial height were the most appropriate levels for predicting mean brightness in the hybrid and mean basic density in the American clone, respectively.
\end{abstract}

Key words: sampling height, willow, density, brightness, Delta of Paraná.

\section{RESUMEN}

El objetivo de este trabajo fue ubicar, dentro de la porción útil del tronco, el punto de muestreo óptimo para la evaluación de densidad básica y blancura, en dos clones de Salix implantados en dos sitios productivos del Delta del Paraná, Argentina. Para ello se estudiaron 32 árboles, de entre 8 y 13 años de edad. De cada uno se obtuvieron rodajas a tres alturas sobre el fuste $(1,30 \mathrm{~m}, 25 \%$ y $65 \%$ de la altura comercial), constituyendo las "secciones predictoras". En cada una de ellas se determinó densidad básica y blancura según dos orientaciones cardinales y tres posiciones en el radio (33\%, 66\% y 100\% de la longitud del radio), totalizando 18 posiciones de muestreo por árbol. La estimación de los parámetros densidad básica y blancura adjudicada al árbol completo y secciones predictoras fue calculada como promedios ponderados. Se estudió la relación entre las estimaciones de densidad y blancura del árbol completo y los valores en cada una de las alturas muestreadas, mediante el análisis de regresión lineal. Considerando el nivel de ajuste logrado, la altura del pecho $(1,30 \mathrm{~m})$ resultó ser la más apropiada para predecir los valores medios de densidad en sauce americano y blancura en sauce híbrido, correspondientes al árbol entero. Los niveles de $25 \%$ y $65 \%$ de la altura comercial presentaron los mejores ajustes para la predicción de la blancura promedio en el híbrido y la densidad promedio en el americano, respectivamente.

Palabras clave: altura de muestreo, sauce, densidad, blancura, Delta del Paraná. 


\section{INTRODUCCION}

El Delta del río Paraná, en Argentina, abarca 1.750.000 ha de islas de origen aluvional, sometidas a periódicas inundaciones. Los suelos son particularmente aptos para el cultivo de Salicáceas, conformando la mayor superficie del mundo cultivada con especies del género Salix L. (sauces) (1).

Según la Secretaría de Agricultura, Ganadería, Pesca y Alimentación (2) se comercializaron $65.000 \mathrm{t}$ de rollizos de sauce en el Delta en el año 2001, destinadas prácticamente en su totalidad a la industria celulósico-papelera, abasteciendo la planta de celulosa de fibra corta para papel periódico más importante del país.

El concepto de aptitud papelera involucra una serie de parámetros relacionados con el leño, con la pasta y con el papel, entre ellos, la densidad básica y la "blancura" o blancura de la madera.

La densidad básica se asocia con los rendimientos del proceso industrial, las resistencias del papel y su opacidad.

La blancura cobra especial importancia cuando la pulpa es obtenida a través de procesos de alto rendimiento. Debido a que sólo una pequeña parte de los componentes responsables del color son eliminados, el blancura de la pulpa obtenida depende en gran medida de la materia prima de la que se partió. Existen muchos antecedentes sobre la medición de este parámetro en pulpa y/o papel, no así en madera. En la selección de los productos del mejoramiento genético, la determinación del blancura sobre la materia prima sin procesar implica un importante beneficio en términos de costos y tiempos, respecto de los procedimientos que requieren el procesamiento previo hasta llegar a la pulpa.

Es una práctica frecuente la utilización de muestras de madera obtenidas a la altura del pecho $(\mathrm{AP}=1,30 \mathrm{~m})$, como representativas para la evaluación de parámetros del árbol completo. Sin embargo, la existencia de variaciones importantes en los valores de densidad y blancura dentro de los árboles hace necesario ubicar un nivel de muestreo adecuado, desde el cual obtener muestras mediante métodos no destructivos, que permitan caracterizar a los árboles en términos de su densidad o blancura.
Según Downes et al. (3), el punto de extracción de la muestra debería cumplir con algunas consideraciones:

- ser fácilmente alcanzado desde el suelo

- otorgar una alta correlación $(r>0,7)$ entre los valores obtenidos a una cierta altura y los del árbol entero

- ser estable ante cambios en la variación médula-corteza, entre y dentro de árboles y a través de diferentes sitios y edades.

No siempre pueden cumplirse todas estas condiciones, principalmente aquellas que consideran que la altura ideal de muestreo debe ser sólida a través de sitios y edades. Busnardo et al. $(4,5)$ consideran que las ecuaciones matemáticas que relacionan los valores a una altura dada con el promedio del árbol deben ser restringidas a determinadas condiciones (de especie, clon, sitio, edad).

Existen importantes antecedentes para el género Eucalyptus L'Heritier sobre la ubicación del punto ideal de muestreo para evaluación de calidad de madera desde un punto de vista celulósicopapelero. Es así que Ferreira (6), Sardinha y Hughes (7), Bonavía de Guth (8), Jorge et al. (9) e Igartúa et al. (10) analizaron la precisión que brindan las valoraciones obtenidas a 1,30 m como predictoras de la densidad y longitud de fibras promedio del árbol completo. Otros autores evaluaron además otras alturas de muestreo, distintas a la mencionada, y en muchos casos resultaron mejores predictoras que la $\mathrm{AP}(3,4,5,11,12,13$, $14,15,16)$.

La estimación de los parámetros adjudicada tanto al árbol completo como a una sección predictora fue calculada, en los trabajos mencionados, como promedios simples o ponderados.

No se han hallado antecedentes referidos al género Salix.

El objetivo de este trabajo fue ubicar, dentro de la porción útil del tronco, el punto de muestreo óptimo para la evaluación de densidad básica y blancura, en dos clones de Salix implantados en dos sitios productivos del Delta del Paraná (Argentina).

Hipótesis de trabajo: existe una sección a cierta altura del árbol en la cual la representatividad es suficientemente significativa como para constituirse en predictora de los valores de densidad y blancura del árbol entero. 


\section{MATERIAL Y METODOS}

Los individuos muestreados pertenecían a dos sitios de producción ubicados en el establecimiento "Las Carabelas", Bajo Delta, provincia de Buenos Aires, Argentina (34³0' S; 59 ${ }^{\circ} 00^{\prime} \mathrm{W}$ ):

1. Dentro de dique: este sitio se encuentra protegido de las inundaciones por un dique de 5,50 m de cota y cuenta con sistemas de bombeo para el desalojo rápido del agua que ocasionalmente pueda afectarlo. Los suelos han sido cultivados los últimos 70 años con frutales y álamos principalmente, con y sin manejo.

2. Fuera de dique: el terreno cuenta con un dique de menor cota $(3,50 \mathrm{~m})$, siendo afectado por inundaciones recurrentes, en las que el tiempo de evacuación del agua está condicionado por las sudestadas del Río de La Plata. Los suelos son prácticamente vírgenes, siendo ésta la primera rotación de cultivos de Salix.

Los clones utilizados fueron: Salix babylonica var. sacramenta $\mathrm{H}$. (sauce americano) y el sauce híbrido Salix babylonica L. x Salix alba L. cv. A131-25, ambos de amplia difusión de cultivo y utilizados como progenitores en programas de mejoramiento genético. Se seleccionaron ocho árboles por clon en cada uno de los dos sitios de producción. Los ejemplares de sauce americano pertenecían a plantaciones de 11 y 13 años de edad y los del sauce híbrido a plantaciones de 10 y 8 años, en los sitios 1 y 2, respectivamente.

En cada ejemplar se marcó la altura del pecho (AP) a 1,30 m y se procedió al apeo. Se midió la altura comercial (HC), definida por un diámetro mínimo de $7,5 \mathrm{~cm}$, y se marcaron dos alturas proporcionales a la misma, desde el nivel del suelo: $25 \%$ y $65 \%$ de HC. Se retiraron dos discos en cada uno de estos tres niveles de muestreo axial: AP, $25 \%$ y $65 \%$ de $\mathrm{HC}$, denominadas alturas 1,2 y 3 , respectivamente. Uno de los discos se utilizó para las determinaciones de densidad básica y el otro para las de blancura.

En el cuadro 1 se presentan los valores medios de los datos dasométricos de los individuos analizados.

Para la medición de densidad, una de las rodajas extraídas en cada altura de muestreo se descortezó y sobre ambas caras se midieron los diámetros sin corteza. Estos valores se promediaron y la mitad de este resultado se consideró el radio correspondiente a la sección a la altura $\mathrm{h}\left(\mathrm{r}_{\mathrm{h}}\right)$. A continuación las rodajas se dividieron de la siguiente manera: se marcaron cuñas de aproximadamente $20^{\circ}$ según dos direcciones cardinales al azar y se obtuvieron tres probetas de cada cuña ( 1,2 y 3 , en el sentido médula-corteza), proporcionales a la longitud del radio de la rodaja, abarcando cada una un tercio del mismo y sumadas, la totalidad de la cuña. De este modo, vistas en superficie, la probeta 1 presentó forma triangular, mientras que las probetas 2 y 3 mostraron forma de cono truncado. El número de probetas fue de seis por altura, es decir, 18 por árbol. Una vez obtenidas las probetas, se determinó la densidad en cada una de ellas según la norma TAPPI 258-om-94 (17).

Las rodajas para la determinación de blancura se descortezaron y de cada una de ellas se extrajo una tabla de $1,5 \mathrm{~cm}$ de espesor, conteniendo la médula. En cada tabla se efectuaron tres determinaciones a cada lado de la médula, denominadas 1 (próxima a médula), 2 (mitad) y 3 (próxima a corteza), abarcando cada una de ellas un tercio de la longitud del radio.

Las mediciones se realizaron con un reflectómetro Elrepho, de geometría $\mathrm{d} / 0^{\circ}$, iluminante C. Se utilizó el filtro 8 (R457), obteniéndose los valores de reflectancia correspondientes a blancura.

CALCULO DE VALORES MEDIOS CORRESPONDIENTES AL ARBOL COMPLETO Y A LAS SECCIONES "PREDICTORAS"

Basado en estudios previos $(9,10)$, la estimación de los parámetros correspondientes al árbol completo y a las alturas predictoras se resolvió utilizando promedios ponderados.

Para obtener los promedios ponderados de densidad básica y blancura de la sección transversal correspondiente a la altura de muestreo h ( $\mathrm{sec}$ ción predictora), se consideraron como ponderaciones a las áreas de los sectores anulares concéntricos en dicha sección, delimitados por el $33 \%, 66 \%$ y $100 \%$ del radio. Similar esquema fue utilizado por Jorge et al. (9) e Igartúa et al. (10) en Eucalyptus.

$$
P_{h}=\frac{\sum_{i=1}^{3} p h i \cdot A_{h i}}{\sum_{i=1}^{3} A_{h i}}
$$




\section{CUADRO 1}

Valores medios de DAP (diámetro a la altura del pecho) (m), altura comercial (m) y altura total $(\mathrm{m})$ de los árboles seleccionados en los sitios 1 y 2.

DBH (diameter at breast height) $(\mathrm{m})$, commercial height $(\mathrm{m})$ and total height $(\mathrm{m})$ mean values of selected trees in sites 1 and 2 .

\begin{tabular}{|c|c|c|c|c|c|}
\hline Clon & Sitio & Arbol & $\begin{array}{l}\text { Altura total } \\
\text { (m) }\end{array}$ & $\begin{array}{l}\text { Altura comercial } \\
\text { (m) }\end{array}$ & DAP $(\mathrm{m})$ \\
\hline \multirow{16}{*}{$\begin{array}{c}\text { Sauce } \\
\text { americano }\end{array}$} & \multirow{8}{*}{1} & 1 & 17,90 & 13,25 & 0,1865 \\
\hline & & 2 & 17,39 & 12,26 & 0,1750 \\
\hline & & 3 & 17,18 & 12,63 & 0,2050 \\
\hline & & 4 & 16,29 & 11,06 & 0,1250 \\
\hline & & 5 & 17,20 & 11,59 & 0,1600 \\
\hline & & 6 & 16,82 & 11,24 & 0,1635 \\
\hline & & 7 & 16,04 & 10,26 & 0,1475 \\
\hline & & 8 & 17,56 & 12,48 & 0,1770 \\
\hline & \multirow{8}{*}{2} & 21 & 17,40 & 12,60 & 0,1810 \\
\hline & & 22 & 18,90 & 13,30 & 0,1030 \\
\hline & & 23 & 18,75 & 13,05 & 0,2055 \\
\hline & & 24 & 18,25 & 13,10 & 0,1815 \\
\hline & & 25 & 16,30 & 10,50 & 0,2030 \\
\hline & & 26 & 18,95 & 13,87 & 0,1290 \\
\hline & & 27 & 17,44 & 12,85 & 0,1545 \\
\hline & & 28 & 15,30 & 6,35 & 0,1075 \\
\hline \multirow{16}{*}{$\begin{array}{l}\text { Sauce } \\
\text { híbrido }\end{array}$} & \multirow{8}{*}{1} & 11 & 15,32 & 10,75 & 0,1455 \\
\hline & & 12 & 16,15 & 10,95 & 0,1520 \\
\hline & & 13 & 16,45 & 10,40 & 0,1665 \\
\hline & & 14 & 15,40 & 9,10 & 0,1235 \\
\hline & & 15 & 15,80 & 10,45 & 0,1460 \\
\hline & & 16 & 15,05 & 9,20 & 0,1240 \\
\hline & & 17 & 14,96 & 9,90 & 0,1420 \\
\hline & & 18 & 15,35 & 10,10 & 0,1510 \\
\hline & \multirow{8}{*}{2} & 31 & 11,60 & 5,80 & 0,1240 \\
\hline & & 32 & 13,50 & 8,20 & 0,1195 \\
\hline & & 33 & 12,45 & 7,30 & 0,1290 \\
\hline & & 34 & 12,68 & 9,90 & 0,1160 \\
\hline & & 35 & 11,88 & 5,48 & 0,0940 \\
\hline & & 36 & 10,72 & 5,34 & 0,1045 \\
\hline & & 37 & 11,62 & 6,73 & 0,1130 \\
\hline & & 38 & 9,36 & 2,45 & 0,0840 \\
\hline
\end{tabular}

$\mathrm{P}_{\mathrm{h}}$ : promedio ponderado del parámetro en la sección transversal a la altura $h$.

i : posición radial $(i=1,2,3)$.

$\mathrm{p}_{\mathrm{hi}}$ : valor del parámetro en la posición radial i a la altura $\mathrm{h}$ (promedio de las determinaciones en las 2 direcciones cardinales).
$\mathrm{A}_{\mathrm{hi}}$ : área del sector anular que contiene a la posición radial i.

El área $A_{\text {hi }}$ se calculó de acuerdo a:

$$
A_{h i}=\pi \cdot r_{h}^{2} \cdot c_{1}
$$


$\mathrm{r}_{\mathrm{h}}=$ radio de la rodaja o sección a la altura $\mathrm{h}$.

$c_{i}=$ coeficiente que depende de los porcentajes de $\mathrm{r}_{\mathrm{h}}$ que delimitan cada sector anular. Para este caso hay tres: $c_{1}=0,33^{2} ; c_{2}=0,66^{2}-$ $0,33^{2}$ y c $_{3}=1-0,66^{2}$.

Como $\pi \cdot \mathrm{r}_{\mathrm{h}}{ }^{2}$ es constante para una misma altura h, la expresión [1] puede simplificarse como sigue:

$$
\begin{aligned}
P_{h}=\frac{\sum_{i=1}^{3} p_{h i} \cdot c_{i}}{\sum_{i=1}^{3} \cdot c_{i}} \\
\text { y como } \sum_{i=1}^{3} \cdot c_{i}=1 \\
P_{h}=\sum_{i=1}^{3} p_{h i} \cdot c_{i}
\end{aligned}
$$

Así, la expresión [4] demuestra que la ponderación del parámetro de interés en cualquier sección transversal h no depende de la magnitud de su diámetro o radio, sino de los porcentajes de radio que delimitan cada sector anular.

Para obtener el promedio ponderado del parámetro de interés para el árbol completo (P), se ponderaron los promedios de las secciones transversales $\left(\mathrm{P}_{\mathrm{h}}\right)$ con volúmenes de secciones cónicas apropiadas, según:

$$
P=\frac{P_{1} \cdot V_{1}+\sum_{h=2}^{3}\left(\left(P_{h}+P_{h-1}\right) / 2\right) \cdot V_{h}}{\sum_{h=1}^{3} V h}
$$

$\mathrm{P}_{\mathrm{h}}$ : promedio ponderado del parámetro en la sección transversal a la altura $h$.

$\mathrm{h}$ : altura de muestreo, $\mathrm{h}=1,2,3(1=\mathrm{AP}=$ $1,30 \mathrm{~m} ; 2=25 \%$ de $\mathrm{HC} ; 3=65 \%$ de $\mathrm{HC}$ ).

$\mathrm{Vh}$ : volumen del cono truncado de radio $\mathrm{r}_{\mathrm{h}}$ en la base superior, radio $\mathrm{r}_{\mathrm{h}-1}$ en la base inferior y altura $a_{h}$.

El volumen del cono truncado (excluyendo la expresión constante $\pi / 3$ ) resultó de la ecuación:

$$
V_{h}=a_{h}\left(r_{h}^{2}+r_{h} \cdot r_{h-1}+r_{h-1}^{2}\right)
$$

Las alturas $\mathrm{a}_{\mathrm{h}}$ de los tres conos definidos dependen de las alturas de muestreo sobre el árbol; en este caso son:

$\mathrm{a}_{1}=1 \mathrm{~m}$ (base superior: AP; base inferior: 0,30 $\mathrm{m})$

$\mathrm{a}_{2}=0,25 \mathrm{HC}-1,30 \mathrm{~m}$ (base superior: $25 \% \mathrm{HC}$; base inferior: AP)

$\mathrm{a}_{3}=0,65 \mathrm{HC}-\mathrm{a}_{2}-1,30 \mathrm{~m}=0,40 \mathrm{HC}$ (base superior: $65 \% \mathrm{HC}$; base inferior: $25 \% \mathrm{HC}$ )

La excepción fue uno de los árboles del sauce híbrido, en el cual la segunda altura $(25 \% \mathrm{HC})$ se ubicó por debajo de AP, por lo tanto las fórmulas se modificaron como se expresa a continuación: $\mathrm{a}_{1}=0,30 \mathrm{~m}$ (base superior: $25 \% \mathrm{HC}$; base inferior: $0,30 \mathrm{~m}$ )

$\mathrm{a}_{2}=1,30 \mathrm{~m}-0,25 \mathrm{HC}$ (base superior: AP; base inferior: $25 \% \mathrm{HC}$ )

$\mathrm{a}_{3}=0,65 \mathrm{HC}-1,30 \mathrm{~m}$ (base superior: $65 \% \mathrm{HC}$; base inferior: AP)

Se procedió a analizar las asociaciones entre los valores de densidad y blancura del árbol completo y los valores de dichos atributos en cada una de las alturas de muestreo estudiadas. Se utilizó para ello el análisis de regresión lineal.

Las ecuaciones que presentaron los mejores ajustes fueron validadas a partir de datos provenientes de otros ocho árboles ubicados en las mismas plantaciones. Para ello se cortaron dos individuos por clon y sitio y se extrajeron discos en las mismas tres alturas de muestreo mencionadas.

En cada rodaja se realizaron 18-20 mediciones de blancura y se determinó la densidad básica de toda la pieza. Estos valores se ponderaron por los volúmenes apropiados para obtener la blancura y densidad del árbol completo (valores observados). Los valores esperados se calcularon a partir de las ecuaciones de regresión, reemplazando $x$ por el valor de la variable a la altura correspondiente según el clon.

Se efectuaron pruebas de comparación de medias ( $t$ de Student) entre los valores observados y esperados, intentando detectar posibles diferencias. 


\section{RESULTADOS Y DISCUSION}

Densidad. Se presentan en los cuadros 2 y 3 los valores medios de densidad básica para el árbol completo y las secciones predictoras, en sauce americano e híbrido, respectivamente.

Los ajustes desarrollados entre la media del árbol y las medias de las alturas de muestreo fueron los presentados en el cuadro 4.

Al utilizar promedios ponderados de densidad (cuadro 4), los ajustes que resultaron mejores para sauce americano fueron los obtenidos a partir de la densidad al $25 \% \mathrm{HC}$ en el sitio $1\left(\mathrm{R}^{2}=81,6 \%\right)$ y AP en el sitio $2\left(\mathrm{R}^{2}=62,2 \%\right)$. Cuando ambos sitios se consideraron juntos, fue la densidad obtenida a $1,30 \mathrm{~m}$ la que nuevamente presentó la mejor relación con la densidad media del árbol $\left(\mathrm{R}^{2}=80,3 \%\right)$, superando el ajuste obtenido en el sitio 2 .
El sauce híbrido presentó los mejores ajustes con las densidades obtenidas como promedios ponderados al $65 \% \mathrm{HC}$ para el sitio $1\left(\mathrm{R}^{2}=\right.$ $75,6 \%)$, AP para el sitio $2\left(\mathrm{R}^{2}=95,5 \%\right)$ y $65 \%$ HC para ambos sitios $\left(R^{2}=87,4 \%\right)$.

La AP, que constituye el nivel más comúnmente usado para extraer muestras de madera que permitan evaluar parámetros del árbol completo, resultó una muy buena "altura predictora" para el sauce híbrido en el sitio 2 y el sauce americano en los sitios 1 y 2 juntos (cuadro 4). Considerando los buenos ajustes logrados al analizar ambos sitios juntos, se recomienda la utilización de la ecuación siguiente para sauce americano:

$$
\begin{aligned}
& * * y\left(\mathrm{~kg} \mathrm{~m}^{-3}\right)=135,4+0,6950 x_{1} \\
& \left(\mathrm{R}^{2}=80,3 \% ; \mathrm{r}=0,90\right)
\end{aligned}
$$

La figura 1 ilustra la regresión anterior.

\section{CUADRO 2}

Valores medios ponderados de densidad básica $\left(\mathrm{kg} \mathrm{m}^{-3}\right)$ por altura de muestreo y para el árbol completo, correspondientes a los individuos de sauce americano.

Weighted mean values of basic density $\left(\mathrm{kg} \mathrm{m}^{-3}\right)$ at sampling heights and for the whole tree, corresponding to American willow trees.

\begin{tabular}{|cccccc|}
\hline \multirow{2}{*}{ Sitio } & N ${ }^{\circ}$ individuo & AP & $25 \%$ HC & $65 \%$ HC & $\begin{array}{c}\text { Arbol } \\
\text { completo }\end{array}$ \\
\hline & 1 & 445,6 & 448,8 & 444,8 & 446,6 \\
& 2 & 433,5 & 443,6 & 443,8 & 439,8 \\
& 3 & 447,7 & 450,1 & 431,4 & 444,7 \\
& 4 & 434,6 & 434,0 & 439,6 & 435,7 \\
1 & 5 & 421,8 & 447,2 & 463,4 & 442,0 \\
& 6 & 434,7 & 434,1 & 434,8 & 434,5 \\
& 7 & 422,3 & 439,6 & 434,6 & 431,5 \\
& 8 & 424,2 & 425,6 & 443,4 & 429,3 \\
& Promedio & 433,0 & 440,4 & 442,0 & 438,0 \\
\hline 21 & 433,9 & 431,1 & 433,2 & 432,8 \\
& 22 & 415,6 & 422,0 & 447,4 & 429,3 \\
& 23 & 398,0 & 431,5 & 423,2 & 416,2 \\
& 24 & 412,5 & 420,1 & 424,8 & 418,2 \\
& 25 & 403,6 & 433,5 & 420,8 & 418,7 \\
& 26 & 413,0 & 430,0 & 405,5 & 417,1 \\
& 27 & 398,8 & 414,7 & 425,5 & 410,8 \\
& 28 & 408,0 & 422,2 & 394,9 & 408,6 \\
& Promedio & 410,4 & 425,6 & 421,9 & 419,0 \\
\end{tabular}




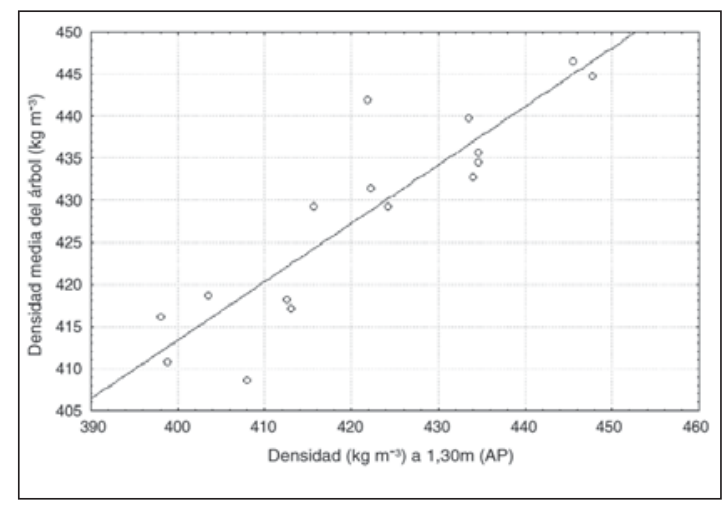

Figura 1. Gráfico de dispersión y recta de regresión estimada para la densidad básica promedio del árbol vs. la densidad básica a 1,30 m. Estimaciones obtenidas como promedios ponderados para sauce americano en los sitios 1 y 2 .

Scatterplot and line of regression estimated for mean basic density of the whole tree vs. basic density at $1.30 \mathrm{~m}$. Estimations obtained as weighted mean values for American willow in sites 1 and 2 .
Por otra parte, el 65\% HC resultó con mejores ajustes para el sauce híbrido en el sitio 1 y cuando ambos sitios fueron analizados juntos (cuadro 4). Se recomienda entonces elegir este nivel para sauce híbrido, que dio para ambos sitios en conjunto el ajuste obtenido a partir de medias ponderadas que se presenta a continuación:

$$
\begin{aligned}
& * * y\left(\mathrm{~kg} \mathrm{~m}^{-3}\right)=180,2+0,5330 x_{3} \\
& \left(\mathrm{R}^{2}=87,36 \% ; \mathrm{r}=0,93\right)
\end{aligned}
$$

La figura 2 grafica la ecuación precedente.

Las ecuaciones [7] y [8] se validaron a partir de datos provenientes de ocho árboles diferentes a los utilizados para su elaboración, pero implantados en los mismos sitios. Para sauce americano, la comparación de medias arrojó valores de $\mathrm{t}=0,167$ y $\mathrm{p}=0,872$, indicando que no existen diferencias significativas entre los valores medios de densidad observados y los calculados a partir de la ecuación [7]. En sauce híbrido se encontró

\section{CUADRO 3}

Valores medios ponderados de densidad básica $\left(\mathrm{kg} \mathrm{m}^{-3}\right)$ por altura de muestreo y para el árbol completo, correspondientes a los individuos de sauce híbrido.

Weighted mean values of basic density $\left(\mathrm{kg} \mathrm{m}^{-3}\right)$ at sampling heights and for the whole tree,

\begin{tabular}{|c|c|c|c|c|c|}
\hline Sitio & $\mathrm{N}^{\circ}$ individuo & AP & $25 \% \mathrm{HC}$ & $65 \% \mathrm{HC}$ & $\begin{array}{c}\text { Arbol } \\
\text { completo }\end{array}$ \\
\hline \multirow{8}{*}{1} & 11 & 394,1 & 371,5 & 370,4 & 380,0 \\
\hline & 12 & 392,1 & 382,4 & 410,6 & 393,2 \\
\hline & 13 & 389,8 & 387,1 & 391,1 & 389,2 \\
\hline & 14 & 378,5 & 372,4 & 371,0 & 374,2 \\
\hline & 15 & 379,0 & 379,2 & 375,6 & 378,2 \\
\hline & 16 & 388,1 & 388,0 & 370,8 & 383,4 \\
\hline & 17 & 384,1 & 373,5 & 369,9 & 377,1 \\
\hline & 18 & 388,8 & 389,2 & 382,3 & 387,2 \\
\hline \multirow{10}{*}{2} & Promedio & 386,8 & 380,4 & 380,2 & 382,8 \\
\hline & 31 & 408,2 & 404,8 & 427,9 & 412,7 \\
\hline & 32 & 401,0 & 399,2 & 414,1 & 404,1 \\
\hline & 33 & 403,4 & 381,4 & 426,9 & 402,1 \\
\hline & 34 & 392,4 & 393,4 & 412,8 & 397,8 \\
\hline & 35 & 389,8 & 395,2 & 395,8 & 392,5 \\
\hline & 36 & 389,3 & 393,8 & 380,5 & 388,1 \\
\hline & 37 & 391,4 & 401,0 & 401,4 & 397,4 \\
\hline & 38 & 368,4 & 363,4 & 365,9 & 366,4 \\
\hline & Promedio & 393,0 & 391,5 & 403,2 & 395,1 \\
\hline
\end{tabular}
corresponding to hybrid willow trees. 


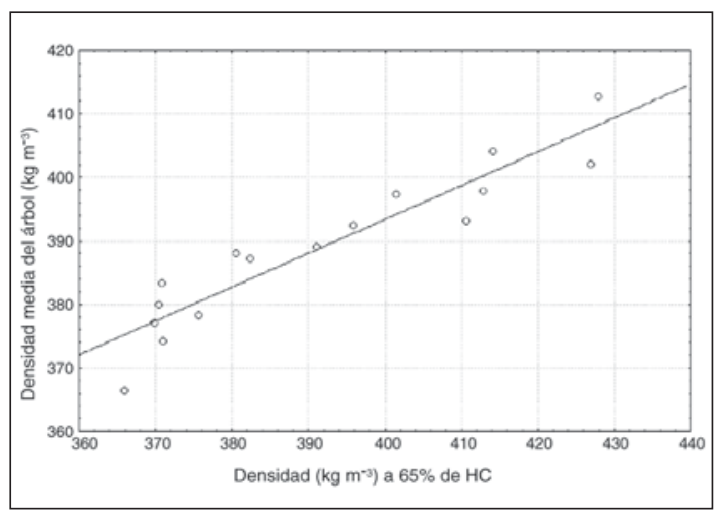

Figura 2. Gráfico de dispersión y recta de regresión estimada para la densidad básica promedio del árbol vs. la densidad básica a $65 \%$ de la altura comercial. Estimaciones obtenidas como promedios ponderados para sauce híbrido en los sitios 1 y 2 .

Scatterplot and line of regression estimated for mean basic density of the whole tree vs. basic density at $65 \%$ of commercial height. Estimations obtained as weighted mean values for hybrid willow in sites 1 and 2 . un resultado similar, con valores de $\mathrm{t}=-0,266 \mathrm{y}$ $\mathrm{p}=0,799$.

Si bien no se han hallado estudios similares para sauce, varios autores han desarrollado trabajos semejantes en Eucalyptus, obteniendo resultados que concuerdan con los de este estudio.

Algunos han encontrado buenos ajustes entre la densidad media del árbol (obtenida ya sea como promedio simple o ponderado) y la densidad media a la $\operatorname{AP}(6,7,8,10,11)$. Jorge et al. (9) halló resultados similares para longitud de fibras.

Otros autores, también para Eucalyptus, hallaron mejores predicciones para la densidad media del árbol a partir del 25\% HC (4), 30\% HC (12) o $50 \%$ HC (5). En nuestro caso, el nivel de $25 \%$ $\mathrm{HC}$ dio buenos ajustes para sauce americano en el sitio 1.

Para Eucalyptus globulus y Eucalyptus nitens, autores como Downes et al. (3), Raymond et al. (13), Muneri y Raymond (14), Raymond y Muneri (15) analizaron alturas fijas como posibles puntos

\section{CUADRO 4}

Modelos predictivos para densidad básica $\left(\mathrm{kg} \mathrm{m}^{-3}\right)$ del árbol completo (y), sobre la base de la densidad básica $\left(\mathrm{kg} \mathrm{m}^{-3}\right)$ a la altura del pecho $\left(\mathrm{x}_{1}\right) ; 25 \% \mathrm{HC}\left(\mathrm{x}_{2}\right)$ y $65 \% \mathrm{HC}\left(\mathrm{x}_{3}\right)$.

Valores de $\mathrm{y}, \mathrm{x}_{1}, \mathrm{x}_{2}$ y $\mathrm{x}_{3}$ obtenidos como promedios ponderados.

Predictive models for basic density $\left(\mathrm{kg} \mathrm{m}^{-3}\right)$ for the whole tree $(\mathrm{y})$, based on basic density $\left(\mathrm{kg} \mathrm{m}^{-3}\right)$ determined at breast height $\left(\mathrm{x}_{1}\right) ; 25 \%$ of commercial height $\left(\mathrm{x}_{2}\right)$ and $65 \%$ of commercial height $\left(\mathrm{x}_{3}\right)$

\begin{tabular}{|c|c|c|c|c|}
\hline Sitio & Clon & Ajuste & $\mathrm{R}^{2}(\%)$ & $\mathrm{r}$ \\
\hline \multirow{3}{*}{1} & Sauce americano & $\mathrm{y}\left(\mathrm{kg} \mathrm{m}^{-3}\right)=149,3+0,6555 \mathrm{x}_{2}$ & 81,61 & $0,90 * *$ \\
\hline & Sauce & $\mathrm{y}\left(\mathrm{kg} \mathrm{m}^{-3}\right)=134,4+0,6530 \mathrm{x}_{2}$ & 52,81 & $0,73 *$ \\
\hline & híbrido & $\mathrm{y}\left(\mathrm{kg} \mathrm{m}^{-3}\right)=231,0+0,3991 \mathrm{x}_{3}$ & 75,64 & $0,87 * *$ \\
\hline \multirow{5}{*}{2} & Sauce & $\mathrm{y}\left(\mathrm{kg} \mathrm{m}^{-3}\right)=186,2+0,5671 \mathrm{x}_{1}$ & 62,16 & $0,79 *$ \\
\hline & americano & $\mathrm{y}\left(\mathrm{kg} \mathrm{m}^{-3}\right)=256,5+0,3849 \mathrm{x}_{3}$ & 55,22 & $0,74 *$ \\
\hline & \multirow{3}{*}{$\begin{array}{c}\text { Sauce } \\
\text { híbrido }\end{array}$} & \multirow{3}{*}{$\begin{array}{l}\mathrm{y}\left(\mathrm{kg} \mathrm{m}^{-3}\right)=-41,1+1,1100 \mathrm{x}_{1} \\
\mathrm{y}\left(\mathrm{kg} \mathrm{m}^{-3}\right)=66,4+0,8395 \mathrm{x}_{2} \\
\mathrm{y}\left(\mathrm{kg} \mathrm{m}^{-3}\right)=157,7+0,5887 \mathrm{x}_{3}\end{array}$} & 95,48 & $0,98 * *$ \\
\hline & & & 65,47 & $0,81^{*}$ \\
\hline & & & 87,00 & $0,93 * *$ \\
\hline \multirow{6}{*}{1 у 2} & \multirow{3}{*}{$\begin{array}{c}\text { Sauce } \\
\text { americano }\end{array}$} & \multirow{3}{*}{$\begin{array}{l}\mathrm{y}\left(\mathrm{kg} \mathrm{m}^{-3}\right)=135,4+0,6950 \mathrm{x}_{1} \\
\mathrm{y}\left(\mathrm{kg} \mathrm{m}^{-3}\right)=21,4+0,9401 \mathrm{x}_{2} \\
\mathrm{y}\left(\mathrm{kg} \mathrm{m}^{-3}\right)=183,3+0,5677 \mathrm{x}_{3}\end{array}$} & 80,27 & $0,90 * *$ \\
\hline & & & 68,29 & $0,83 * *$ \\
\hline & & & 59,82 & $0,77 * *$ \\
\hline & \multirow{3}{*}{$\begin{array}{l}\text { Sauce } \\
\text { híbrido }\end{array}$} & \multirow{3}{*}{$\begin{array}{l}y\left(\mathrm{~kg} \mathrm{~m}^{-3}\right)=-60,6+1,1529 \mathrm{x}_{1} \\
\mathrm{y}\left(\mathrm{kg} \mathrm{m}^{-3}\right)=53,5+0,8692 \mathrm{x}_{2} \\
\mathrm{y}\left(\mathrm{kg} \mathrm{m}^{-3}\right)=180,2+0,5330 \mathrm{x}_{3}\end{array}$} & 84,07 & $0,92 * *$ \\
\hline & & & 71,00 & $0,84 * *$ \\
\hline & & & 87,36 & $0,93 * *$ \\
\hline
\end{tabular}

Se incluyen sólo ajustes con pendiente significativa $*(\mathrm{p}<0,05)$ y altamente significativa $* *(\mathrm{p}<0,01)$. $\mathrm{Se}$ indican en negrita los ajustes con mayor $\mathrm{R}^{2}$. 
de extracción de las muestras, logrando los mejores resultados al muestrear entre 0,7 y $1,5 \mathrm{~m}$ desde el nivel del suelo, para la evaluación de densidad básica y longitud de fibras.

El efecto del sitio en la obtención de la "altura predictora" podría atribuirse a los diferentes patrones de variación axial de la densidad propios de cada combinación clon-sitio. Estos resultados pueden encontrarse en Villegas y Marlats (18). De todas maneras, puede observarse en el cuadro 2 que para sauce americano la variable aumentó progresivamente en sentido vertical dentro del tronco en el sitio 1, mientras que aumentó y posteriormente descendió en el sitio 2. En el sauce híbrido, en cambio, la densidad básica en el sitio 1 disminuyó entre los dos primeros niveles de muestreo para luego mantenerse estable hacia el ápice, mientras que en el segundo sitio se produjo un aumento en la variable a partir del $25 \%$ de $\mathrm{HC}$, luego de un muy leve descenso inicial (cuadro 3).
La densidad básica es una compleja propiedad, que puede variar según la procedencia, sitio, clon, edad, entre árboles y dentro de un mismo ejemplar, tanto en sentido vertical como horizontal. Los disímiles patrones de variación axial señalados podrían haber determinado que las "alturas predictoras" más apropiadas cambien según el sitio y clon analizados.

Raymond et al. (13) encontraron resultados similares en Eucalyptus nitens; los niveles óptimos de muestreo variaron según el sitio estudiado. En cambio, en Eucalyptus globulus las predicciones fueron consistentes a través de los diferentes sitios de implantación. Los patrones de variación longitudinal de la densidad fueron muy semejantes en todas las localidades para la segunda especie y presentaron importantes diferencias según el sitio en Eucalyptus nitens.

Blancura. Se presentan en los cuadros 5 y 6 los valores medios de blancura para el árbol completo y las secciones predictoras.

\section{CUADRO 5}

Valores medios ponderados de blancura (\%) por altura de muestreo y para el árbol completo, correspondientes a los individuos de sauce americano.

Weighted mean values of brightness (\%) at sampling heights and for the whole tree, corresponding to American willow trees.

\begin{tabular}{|c|c|c|c|c|c|}
\hline Sitio & $\mathrm{N}^{\circ}$ individuo & AP & $25 \% \mathrm{HC}$ & $65 \% \mathrm{HC}$ & $\begin{array}{c}\text { Arbol } \\
\text { completo }\end{array}$ \\
\hline \multirow{8}{*}{1} & 1 & 38,26 & 38,54 & 40,54 & 38,92 \\
\hline & 2 & 38,58 & 42,61 & 47,15 & 42,18 \\
\hline & 3 & 33,53 & 37,42 & 39,56 & 36,40 \\
\hline & 4 & 40,83 & 42,74 & 42,61 & 42,03 \\
\hline & 5 & 40,94 & 41,95 & 44,15 & 42,14 \\
\hline & 6 & 40,97 & 40,66 & 46,67 & 42,25 \\
\hline & 7 & 38,18 & 42,05 & 46,37 & 41,60 \\
\hline & 8 & 40,37 & 43,28 & 47,02 & 43,02 \\
\hline \multirow{8}{*}{2} & 21 & 32,55 & 35,98 & 42,22 & 35,92 \\
\hline & 22 & 36,70 & 41,85 & 46,00 & 42,08 \\
\hline & 23 & 34,57 & 38,48 & 46,33 & 38,69 \\
\hline & 24 & 34,96 & 37,82 & 40,37 & 37,27 \\
\hline & 25 & 34,59 & 38,40 & 38,20 & 36,83 \\
\hline & 26 & 38,23 & 40,49 & 45,59 & 40,77 \\
\hline & 27 & 36,67 & 41,74 & 44,99 & 40,44 \\
\hline & 28 & 37,17 & 38,94 & 46,44 & 39,65 \\
\hline \multicolumn{2}{|c|}{ Promedio del clon } & 37,32 & 40,18 & 44,01 & 40,01 \\
\hline
\end{tabular}




\section{CUADRO 6}

Valores medios ponderados de blancura (\%) por altura de muestreo y para el árbol completo, correspondientes a los individuos de sauce híbrido.

Weighted mean values of brightness $(\%)$ at sampling heights and for the whole tree, corresponding to hybrid willow trees.

\begin{tabular}{|cccccc|}
\hline \multirow{2}{*}{ Sitio } & $N^{\circ}$ individuo & AP & $25 \%$ HC & $65 \%$ HC & $\begin{array}{c}\text { Arbol } \\
\text { completo }\end{array}$ \\
\hline & 11 & 49,72 & 53,34 & 52,24 & 51,67 \\
& 12 & 48,05 & 52,45 & 53,35 & 50,90 \\
13 & 43,58 & 47,08 & 42,99 & 44,71 \\
& 14 & 49,39 & 50,36 & 47,69 & 49,28 \\
& 15 & 38,41 & 43,34 & 46,78 & 42,35 \\
& 16 & 45,43 & 51,16 & 51,10 & 49,04 \\
& 17 & 47,57 & 47,17 & 53,04 & 48,74 \\
18 & 37,69 & 44,16 & 44,30 & 41,88 \\
\hline 31 & 46,32 & 50,26 & 53,58 & 49,53 \\
& 32 & 48,06 & 53,10 & 52,26 & 51,00 \\
& 33 & 44,39 & 47,94 & 51,78 & 47,63 \\
& 34 & 45,87 & 48,13 & 50,91 & 47,91 \\
& 35 & 46,55 & 49,42 & 53,56 & 48,85 \\
& 36 & 43,50 & 43,35 & 51,65 & 45,78 \\
& 37 & 48,93 & 45,42 & 49,47 & 47,93 \\
& 38 & 46,95 & 46,17 & 50,64 & 47,05 \\
\hline \multirow{2}{*}{ Promedio del clon } & 45,65 & 48,30 & 50,33 & 47,77 \\
\hline
\end{tabular}

Los ajustes obtenidos entre y (blancura promedio del árbol) y cada una de las variables predictoras $\left(\mathrm{x}_{1}, \mathrm{x}_{2} \mathrm{y} \mathrm{x}_{3}\right)$ fueron los que se muestran en el cuadro 7 .

Puede observarse en el cuadro 7 que, a partir de medias ponderadas, los mejores ajustes para sauce americano fueron los logrados con el promedio de blancura al $25 \%$ de la altura comercial, en cualquiera de los sitios analizados, considerados en forma conjunta o no $\left(\mathrm{R}^{2}=86,81 \%\right.$, sitio 1 ; $\mathrm{R}^{2}=85,68 \%$, sitio $2 ; \mathrm{R}^{2}=88,99 \%$, ambos sitios).

El sauce híbrido, en cambio, mostró los mejores ajustes a partir del promedio de blancura a 1,30 m (AP) para el sitio 1 y ambos sitios juntos $\left(\mathrm{R}^{2}=91,51 \%\right.$ y $82,51 \%$, respectivamente $)$, y a partir del $25 \%$ HC para el sitio $2\left(\mathrm{R}^{2}=90,88 \%\right)$. El ajuste logrado para el sauce híbrido en los dos sitios juntos fue más bajo que el que se obtuvo para cada sitio en forma separada.

Según lo expuesto, es evidente que la mejor "altura predictora" de blancura para el sauce americano resultó el nivel del 25\% HC, que también había presentado buenos ajustes para densidad en el sitio 1, para este mismo clon. La ecuación obtenida a partir de medias ponderadas, para ambos sitios estudiados, es la siguiente:

$$
\begin{aligned}
& * * y(\%)=-0,3007+1,0032 x_{2} \\
& \left(\mathrm{R}^{2}=88,99 \% ; \mathrm{r}=0,94\right)
\end{aligned}
$$

La misma se grafica en la figura 3 .

En el sauce híbrido, el nivel que mostró la mayor relación con el promedio del árbol para blancura fue la AP, ya sea para el sitio 1 o cuando se consideran ambos sitios juntos. La ecuación recomendada es la siguiente, obtenida a partir de promedios ponderados, para ambos sitios:

$$
\begin{aligned}
& * * y(\%)=14,1198+0,7370 x_{1} \\
& \left(\mathrm{R}^{2}=82,51 \% ; \mathrm{r}=0,91\right)
\end{aligned}
$$

Se ilustra la regresión [10] en la figura 4.

Las ecuaciones [9] y [10] también fueron validadas a partir de datos provenientes de los ocho árboles mencionados. Para ambos clones, no se encontraron diferencias estadísticamente signifi- 


\section{CUADRO 7}

Modelos predictivos para blancura (\%) del árbol completo (y), sobre la base de blancura

(\%) a la altura del pecho $\left(\mathrm{x}_{1}\right) ; 25 \% \mathrm{HC}\left(\mathrm{x}_{2}\right)$ y $65 \% \mathrm{HC}\left(\mathrm{x}_{3}\right)$.

Predictive models for brightness (\%) for the whole tree (y), based on brightness $(\%)$ determined at breast height $\left(\mathrm{x}_{1}\right) ; 25 \%$ of commercial height $\left(\mathrm{x}_{2}\right)$ and $65 \%$ of commercial height $\left(\mathrm{x}_{3}\right)$.

\begin{tabular}{|c|c|c|c|c|}
\hline Sitio & Clon & Ajuste & $\mathrm{R}^{2}(\%)$ & $\mathrm{r}$ \\
\hline \multirow{6}{*}{1} & \multirow{3}{*}{ Sauce americano } & \multirow{3}{*}{$\begin{array}{l}\mathrm{y}(\%)=10,1524+0,7936 \mathrm{x}_{1} \\
\mathrm{y}(\%)=0,6398+0,9823 \mathrm{x}_{2} \\
\mathrm{y}(\%)=13,2387+0,6288 \mathrm{x}_{3}\end{array}$} & 79,07 & $0,89 * *$ \\
\hline & & & 86,81 & $0,93 * *$ \\
\hline & & & 72,92 & $0,85^{* *}$ \\
\hline & \multirow{3}{*}{ Sauce híbrido } & \multirow{3}{*}{$\begin{array}{l}\mathrm{y}(\%)=12,6586+0,7706 \mathrm{x}_{1} \\
\mathrm{y}(\%)=0,7830+0,9570 \mathrm{x}_{2} \\
\mathrm{y}(\%)=9,5561+0,7717 \mathrm{x}_{3}\end{array}$} & 91,51 & $0,96^{* *}$ \\
\hline & & & 88,98 & $0,94 * *$ \\
\hline & & & 67,37 & $0,82 *$ \\
\hline \multirow{4}{*}{2} & \multirow{3}{*}{ Sauce americano } & \multirow{3}{*}{$\begin{array}{l}\mathrm{y}(\%)=3,0675+0,9067 \mathrm{x}_{1} \\
\mathrm{y}(\%)=0,4124+0,9830 \mathrm{x}_{2} \\
\mathrm{y}(\%)=16,2637+0,5185 \mathrm{x}_{3}\end{array}$} & 73,46 & $0,86^{* *}$ \\
\hline & & & 85,68 & $0,92 * *$ \\
\hline & & & 57,00 & $0,76^{*}$ \\
\hline & Sauce híbrido & $\mathrm{y}(\%)=24,326+0,4979 \mathrm{x}_{2}$ & $\mathbf{9 0 , 8 8}$ & $0,95 * *$ \\
\hline \multirow{6}{*}{1 у 2} & \multirow{3}{*}{ Sauce americano } & \multirow{3}{*}{$\begin{array}{l}\mathrm{y}(\%)=10,8921+0,7803 \mathrm{x}_{1} \\
\mathrm{y}(\%)=-0,3007+1,0032 \mathrm{x}_{2} \\
\mathrm{y}(\%)=13,652+0,5989 \mathrm{x}_{3}\end{array}$} & 78,98 & $0,89 * *$ \\
\hline & & & 88,99 & $0,94 * *$ \\
\hline & & & 56,47 & $0,75^{* *}$ \\
\hline & \multirow{3}{*}{ Sauce híbrido } & \multirow{3}{*}{$\begin{array}{l}\mathrm{y}(\%)=14,1198+0,7370 \mathrm{x}_{1} \\
\mathrm{y}(\%)=11,4034+0,7528 \mathrm{x}_{2} \\
\mathrm{y}(\%)=14,4574+0,6617 \mathrm{x}_{3}\end{array}$} & 82,51 & $0,91 * *$ \\
\hline & & & 76,48 & $0,87 * *$ \\
\hline & & & 57,32 & $0,76^{* *}$ \\
\hline
\end{tabular}

Se incluyen sólo ajustes con pendiente significativa $*(\mathrm{p}<0,05)$ y altamente significativa $* *(\mathrm{p}<0,01)$. Se indican en negrita los ajustes con mayor $\mathrm{R}^{2}$.

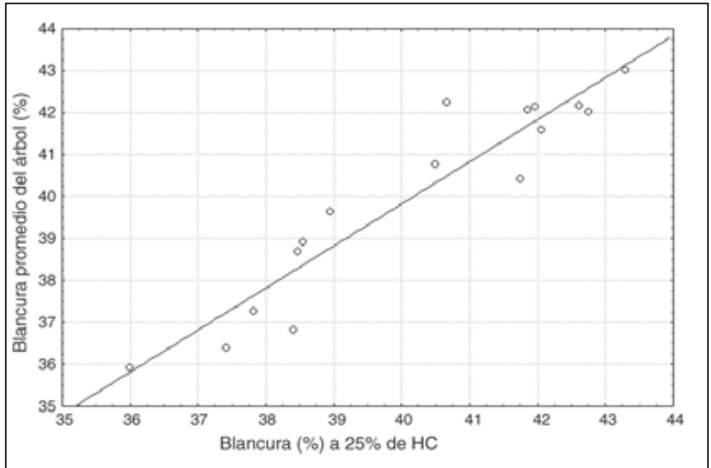

Figura 3. Gráfico de dispersión y recta de regresión estimada para blancura promedio del árbol vs. blancura a $65 \%$ de la altura comercial. Estimaciones obtenidas como promedios ponderados para sauce americano en los sitios 1 y 2 .

Scatterplot and line of regression estimated for mean brightness of the whole tree vs. brightness at $65 \%$ of commercial height. Estimations obtained as weighted mean values for American willow in sites 1 and 2 .

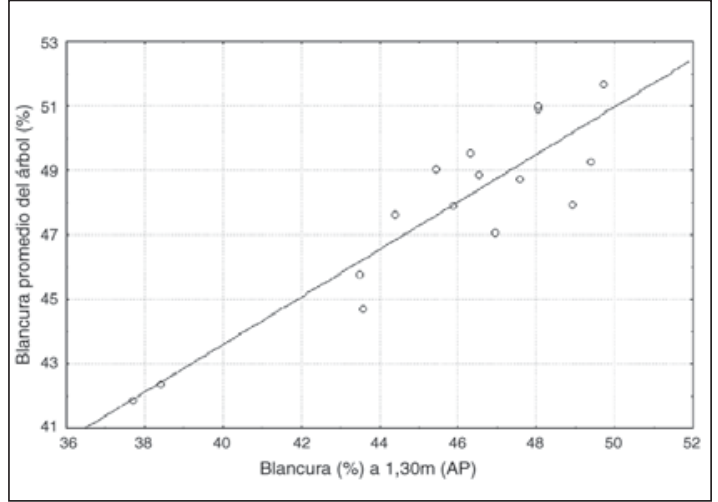

Figura 4. Gráfico de dispersión y recta de regresión estimada para blancura promedio del árbol vs. blancura a $1,30 \mathrm{~m}$. Estimaciones obtenidas como promedios ponderados para sauce híbrido en $\operatorname{los}$ sitios 1 y 2 .

Scatterplot and line of regression estimated for mean brightness of the whole tree vs. brightness at $1.30 \mathrm{~m}$. Estimations obtained as weighted mean values for hybrid willow in sites 1 and 2 . 
cativas entre los valores medios de blancura observados y calculados. Las pruebas de $t$ arrojaron valores de $\mathrm{t}=-0,297, \mathrm{p}=0,777$ en sauce americano y t $=0,017, \mathrm{p}=0,987$ en sauce híbrido.

Raymond et al. (16) no hallaron correlación entre la blancura promedio del árbol y los valores medios obtenidos a partir de muestras de barreno a cuatro alturas fijas, para Eucalyptus nitens. Como el patrón de variación axial de esta propiedad describe un aumento desde la base al ápice, consideraron que el punto ideal de muestreo estaría por encima de los niveles que analizaron, todos ellos inferiores a 1,30 m. Esto queda corroborado al menos para los dos clones de Salix del presente trabajo.

Finalmente, cabe recordar que el uso de las ecuaciones presentadas, tanto para densidad básica como para blancura, debe restringirse a las mismas condiciones planteadas en este estudio. Busnardo et al. (4) concuerdan con esta premisa, indicando que la utilización indiscriminada de este tipo de ecuaciones puede introducir errores en las estimaciones que comprometan los estudios posteriores.

\section{CONCLUSIONES}

- Los valores de densidad básica y blancura del árbol completo y los puntos óptimos de muestreo variaron según los clones, edades y sitios estudiados.

- La representatividad de la muestra tomada a la altura del pecho fue suficientemente significativa como para constituirse en predictora de los valores del árbol entero.

- Teniendo en cuenta las fuentes de variación observadas los modelos aplicados son particulares para el caso estudiado.

\section{BIBLIOGRAFIA}

(1) GOLFARI, L. Condiciones ecológicas del cultivo de las Salicáceas en la Argentina. Revista de Investigaciones Agrícolas, 1958, vol. XII, No 2, p. 173-243.

(2) SECRETARIA DE AGRICULTURA, GANADERIA, PESCA Y ALIMENTACION. Oferta y demanda de ro1lizos según provincia. Año 2001. Area de Economía e Información. Dirección de Forestación.

[Consulta: 5 agosto 2004]. Disponible en http://www.sagpya.mecon.gov.ar/new/0-0/forestacion/ econo/especies01.pdf

(3) DOWNES, G.M., I.L. HUDSON, C.A. RAYMOND, G.H. DEAN, A.J. MICHELL, L.R. SCHIMLECK, R. EVANS, A. MUNERI. Sampling plantation Eucalyptus for wood and fibre properties. Melbourne, Australia: CSIRO publishing, 1997, $132 \mathrm{p}$.

(4) BUSNARDO, C.A., J.V. GONZAGA, C.E.B. FOELKEL C. DIAS, S. MENOCHELLI. Em busca da qualidade ideal da madeira do eucalipto para produção de celulose III. A importancia da altura de amostragem para avaliação da densidade básica média da árvore. III Congresso Latino-Americano de Celulose e Papel, São Paulo, 1985, p. 55-72.

(5) BUSNARDO, C.A., J.V. GONZAGA, C.E.B. FOELKEL, S. MENOCHELLI S. Em busca da qualidade ideal da madeira do eucalipto para produção de celulose IV. Altura ideal de amostragem para avaliação da densidade média para árvores de Eucalyptus grandis. XX Congresso Anual da ABCP-Semana do papel, São Paulo, 1987 , p. $17-33$

(6) FERREIRA, M. Variação da densidade básica da madeira de povoamentos comerciais de Eucalyptus grandis Hill. ex Maiden. nas idades de 11, 12, 13, 14 e 16 anos. IPEF, 1972, vol. 4, p. 65-89.

(7) SARDINHA, R.M. DE A., J.F. HUGHES. Wood properties variation of Eucalyptus saligna Sm. from Angola. Separata do Volume XXXVIII dos Anais do Instituto Superior de Agronomia, 1978-1979, p. 104-124.

(8) BONAVIA DE GUTH, E. Avaliação de seis espécies de eucalipto e apreciação da amostra extraída a altura do peito. III Congresso Latino-Americano de Celulose e Papel, São Paulo, 1985, p. 33-38.

(9) JORGE, F., T. QUILHO, H. PEREIRA. Variability of fibre length in wood and bark in Eucalyptus globulus. IAWA Journal, 2000, vol. 21, $\mathrm{N}^{\circ}$ 1, p. 41-48.

(10) IGARTUA, D.V., S.E. MONTEOLIVA, M.G. MONTERUBBIANESI, M.S. VILLEGAS. Sampling at breast height (bh) level of basic density and fibre length for Eucalyptus globulus ssp. globulus for parameter prediction of the whole tree. IAWA Journal, 2003, vol. 24, $\mathrm{N}^{\circ} 2$, p. 173-184.

(11) BHAT, K.M., K.V. BHAT, T.K. DHAMODARAN. Wood density and fiber length of Eucalyptus grandis grown in Kerala, India. Wood and Fiber Science, 1990, vol. $22, \mathrm{~N}^{\circ} 1$, p. 54-61.

(12) DEAN, G.H., J. FRENCH J., W.N. TIBBITS. Variation in pulp and papermaking characteristics in a field trial of Eucalyptus globulus . 44 $4^{\text {th }}$ Annual General Conference Appita, Rotorua, 1990, Tomo B24, p. 1-33.

(13) RAYMOND, C.A., A. MUNERI, A.C. MACDONALD. Nondestructive sampling for basic density in Eucalyptus globulus and Eucalyptus nitens. Appita Journal, 1998, vol. $51, \mathrm{~N}^{\circ} 3$, p. 224-227.

(14) MUNERI, A., C.A. RAYMOND. Nondestructive sampling of Eucalyptus globulus and Eucalyptus nitens for wood properties. II. Fibre length and coarseness. Wood Science and Technology, 2001, vol. 35, p. 41-56.

(15) RAYMOND, C.A., A. MUNERI. Nondestructive sampling of Eucalyptus globulus and Eucalyptus nitens for wood properties. I. Basic density. Wood Science and Technology, 2001, vol. 35, p. 27-39.

(16) RAYMOND, C.A., A.D. BRADLEY. Assessment of wood colour in Eucalyptus nitens. Appita Journal, 2002, vol. 55, $\mathrm{N}^{\circ} 2$, p. 118-122.

(17) TAPPI T $258 \mathrm{om}-94,1994$. Basic density and moisture content of pulpwood. $8 \mathrm{p}$.

(18) VILLEGAS, M.S., R.M. MARLATS. Densidad de la madera: variación axial y radial en dos clones de Salix $s p$. X Jornadas Técnicas Forestales Ambientales FCFINTA, Eldorado, 2003, x p. 